\title{
The influence of the PRKAG3 mutation on glycogen, enzyme activities and fibre types in different skeletal muscles of exercise trained pigs
}

\author{
Anna Granlund*, Marianne Jensen-Waern and Birgitta Essén-Gustavsson
}

\begin{abstract}
Background: AMP-activated protein kinase (AMPK) plays an important role in the regulation of glucose and lipid metabolism in skeletal muscle. Many pigs of Hampshire origin have a naturally occurring dominant mutation in the AMPK $\gamma 3$ subunit. Pigs carrying this PRKAG3 (R225Q) mutation have, compared to non-carriers, higher muscle glycogen levels and increased oxidative capacity in $\mathrm{m}$. longissimus dorsi, containing mainly type II glycolytic fibres. These metabolic changes resemble those seen when muscles adapt to an increased physical activity level. The aim was to stimulate AMPK by exercise training and study the influence of the PRKAG3 mutation on metabolic and fibre characteristics not only in $\mathrm{m}$. longissimus dorsi, but also in other muscles with different functions.

Methods: Eight pigs, with the PRKAG3 mutation, and eight pigs without the mutation were exercise trained on a treadmill. One week after the training period muscle samples were obtained after euthanisation from $\mathrm{m}$. biceps femoris, $m$. longissimus dorsi, $m$. masseter and $m$. semitendinosus. Glycogen content was analysed in all these muscles. Enzyme activities were analysed on $m$. biceps femoris, $m$. longissimus dorsi, and $m$. semitendinosus to evaluate the capacity for phosphorylation of glucose and the oxidative and glycolytic capacity. Fibre types were identified with the myosin ATPase method and in $m$. biceps femoris and $m$. longissimus dorsi, immunohistochemical methods were also used.

Results: The carriers of the PRKAG3 mutation had compared to the non-carriers higher muscle glycogen content, increased capacity for phosphorylation of glucose, increased oxidative and decreased glycolytic capacity in $m$. longissimus dorsi and increased phosphorylase activity in $m$. biceps femoris and $m$. longissimus dorsi. No differences between genotypes were seen when fibre type composition was evaluated with the myosin ATPase method. Immunohistochemical methods showed that the carriers compared to the non-carriers had a higher percentage of type II fibres stained with the antibody identifying type IIA and IIX fibres in m. longissimus dorsi and a lower percentage of type IIB fibres in both $m$. biceps femoris and $m$. longissimus dorsi. In these muscles the relative area of type IIB fibres was lower in carriers than in non-carriers.
\end{abstract}

Conclusions: In exercise-trained pigs, the PRKAG3 mutation influences muscle characteristics and promotes an oxidative phenotype to a varying degree among muscles with different functions.

\section{Background}

The prevalence of the PRKAG3 mutation in $\mathrm{RN}^{-}$Hampshire pigs has likely been propagated by its favourable effects on the growth rate and on the meat content of the carcass [1,2]. This PRKAG3 mutation is a substitution in the PRKAG3 gene (R225Q), which encodes a

\footnotetext{
* Correspondence: anna.granlund@kv.slu.se

Department of Clinical Sciences, Section for Comparative Physiology and Medicine, Faculty of Veterinary Medicine and Animal Science, Swedish University of Agricultural Sciences, SE-750 07, Uppsala, Sweden
}

muscle specific isoform of the AMP-activated protein kinase (AMPK) $\gamma 3$ subunit expressed mainly in glycolytic muscles in pigs $[3,4]$. AMPK is an energy sensor that is activated by an increase in AMP/ATP ratio and directly phosphorylates many metabolic enzymes and therefore plays an important role in glucose uptake, glycogen synthesis, and fat oxidation in skeletal muscle $[5,6]$. AMPK activation by muscle contraction is a vital step towards exercise-stimulated glucose uptake $[7,8]$. Glycogen will repeatedly be broken down and resynthesised

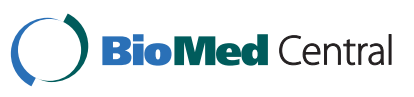


when a muscle is trained which leads to a demand for glucose uptake and activation of AMPK to restore the glycogen used during exercise. Pigs that carry the PRKAG3 mutation have in comparison to non-carriers greater glycogen content and increased oxidative capacity in $m$. longissimus dorsi [4,9]. These metabolic changes resemble those seen in pigs when muscles have adapted to an increased physical activity level $[10,11]$. Few studies have looked into the effect of the PRKAG3 mutations on other skeletal muscles than $m$. longissimus dorsi. Different muscles have different functions within the body, which is reflected by different metabolic and contractile properties of their muscle fibres. For example $m$. masseter is a muscle that is mainly active during the chewing process and $m$. biceps femoris seems to be a muscle that is more active than $m$. semitendinosus and $m$. longissimus dorsi, when pigs are trained on a treadmill $[10,11]$. Contractile characteristics based on different myosin heavy chain (MHC) isoforms differ among fibres and muscles [12]. Hybrid fibres contain more than one MHC isoform and may indicate fibre type transformation. An increased amount of hybrid fibres can be seen in trained muscles of man and rat [13]. The aim of this study was to examine the effect of the PRKAG3 mutation on both the metabolic profile and the fibre characteristics in different muscles ( $m$. longissimus dorsi, $m$. biceps femoris, $m$. semitendinosus and $m$. masseter) after exercise-induced stimulation of AMPK and glycogen metabolism.

\section{Methods}

\section{Animals and housing}

The Ethical Committee for Animal Experiments, Uppsala, Sweden approved of the experimental design.

Sixteen clinically healthy female pigs (Yorkshire/ Swedish Landrace $\times$ Hampshire) at the age of 9-11 weeks and with a mean weight of $29 \pm 0.6 \mathrm{~kg}$ were obtained from the University herd. Eight pigs were heterozygous carriers and eight pigs were non-carriers of the PRKAG3 mutation which was revealed by DNA analyses of blood [3]. All pigs were housed at the department (Department of Clinical Sciences, Swedish University of Agricultural Sciences) in pens with concrete floors and straw as bedding. The animals were fed twice daily ad libitum a commercial finisher diet without growth promoters (Piggfor; Origio 522 PK, Lantmännen, Sweden with an energy content of $12.4 \mathrm{MJ}$ and crude protein content of $13 \%$ ), and had ad libitum access to water. Clinical health examinations were performed daily on all animals throughout the study.

\section{Experimental design}

The protocol ran for nine weeks and started with a two week period of acclimatisation. During this period the pigs also became used to the treadmill (Säto, Knivsta, Sweden). They were allowed to walk and trot on the treadmill for a few minutes on four separate days, before an exercise test was performed and tissue samples from $m$. biceps femoris were obtained by a needle biopsy [14]. Thereafter the pigs trained on the treadmill once daily, five days a week for the next five weeks. The speed continuously increased from $1.5 \mathrm{~m} / \mathrm{s}$ to $2.5 \mathrm{~m} / \mathrm{s}$ and the distance increased from $300 \mathrm{~m}$ to $1000 \mathrm{~m}$. The training period ended with a second exercise test and tissue samples were again obtained from $m$. biceps femoris. Thereafter the pigs had a jugular catheter inserted under general anaesthesia to obtain unstressed blood samples. Also a catheter in situ facilitated a smooth euthanisation and muscle samples were achieved under a minimum of stress. A third exercise test was then performed a week later and tissue samples from $m$. biceps femoris as well as blood samples were obtained. The pigs were then 18 to 20 weeks old and the carriers had a mean weight of $80 \pm 1.5 \mathrm{~kg}$ and the non-carriers had a mean weight $74 \pm 3 \mathrm{~kg}$ with no significant difference between the two genotypes.

Six days after the third exercise test the animals were euthanised by an intravenous infusion of pentobarbital $(100 \mathrm{mg} / \mathrm{mL})$ in their pens. Two pigs were withdrawn from the study after training, one due to unwillingness to run on the treadmill and the other did not survive anaesthesia.

\section{Muscle samples}

Within 10 min after the animals were euthanised, samples of about $2 \times 1 \times 1 \mathrm{~cm}$ were taken from $m$. masseter, $m$. semitendinosus (white portion), m. biceps femoris and $m$. longissimus dorsi (caudal to the last rib) by excision. All muscle specimens were obtained from the centre of the middle part of the muscle. The tissue samples were immediately frozen in liquid nitrogen and stored at minus $80^{\circ} \mathrm{C}$ until analysed. The tissue sample used for histochemistry was rolled in talcum powder before being frozen.

\section{Muscle fibre analyses}

The muscle sample was mounted on embedding medium (OCT compound) and serial transverse sections $(10 \mu \mathrm{m})$ were cut in a cryostat (2800 Frigocut E, Reichert-Jung, Leica Microsystems $\mathrm{GmbH}$ ) at $-20^{\circ} \mathrm{C}$. Myofibrillar ATPase staining with preincubations at $\mathrm{pH} 4.3,4.6$ and 10.3 were used to identify fibre types I, IIA, IIB [15] in all muscles. In $m$. biceps femoris and $m$. longissimus dorsi also immunohistochemical methods were used. Serial sections, were reacted with myosin heavy chain (MHC) antibodies BA-D5 (MHCI) (gift from E.Barrey) and A4-74 (MHCIIA + MHCIIX) (Alexis Biochemicals). The secondary antibody (rabbit anti-mouse immunoglobulins) 
and the peroxidase-anti-peroxidase complex used to visualize the binding to the antibody came from Dako in Denmark. The muscle fibres stained with the antibody A4-74, were classified as IIAX fibres and some of these fibres may be pure IIX and/or IIBX fibres. To evaluate fibre type composition, fibre type area and relative fibre type area, a computerized image analyser (Bio-Rad, Scan Beam, Hadsund, Denmark) was used. One section (containing at least 200 fibres) of the $\mathrm{pH} 4.6$ ATPase stain were photographed and type IIB fibres on this section that corresponded to fibres that stained with the A4-74 antibody were classified as type IIAX fibres. All type I fibres from the ATPase stain corresponded to type I fibres stained with the antibody BA-D5 (MHCI). Sections of $m$. biceps femoris and $m$. longissimus dorsi were also stained with the NADH tetrazolium reductase method [16]. Oxidative capacity was subjectively evaluated from the intensity of the blue staining (30-50 fibres of each type) into high- if the whole fibre was stained, mediumif some staining was apparent, mostly at the cells borders, or low if there was hardly any staining within the cell (Figure 1).

\section{Enzyme activity analyses}

Muscle biopsies were freeze-dried overnight and then muscle tissue was dissected out under a microscope to remove visible blood, fat, and connective tissue. To determine the activities of citrate synthase (CS), 3-hydroxyacyl-CoA dehydrogenase (HAD), lactate dehydrogenase (LDH), hexokinase (HK), and phosphorylase, 1-2 mg of pure tissue was homogenized with an ultrasound disintegrator (Branson) in ice-chilled potassium phosphate buffer $(0.1 \mathrm{M}, \mathrm{pH} 7.3)$ at a dilution of $1: 400$ and then analysed fluorometrically [14,17].

\section{Glycogen analyses}

For glycogen determination 1-2 mg of pure tissue was boiled in $1 \mathrm{M} \mathrm{HCl}$ for $2 \mathrm{~h}$ to form glucose residues. Glucose was analysed with a fluorometric method [17].

\section{Statistical analyses}

Data are presented as means \pm standard errors. For the statistical analyses the values from each genotype were assumed to be independent observations from normal probability distributions. An unpaired t-test was used
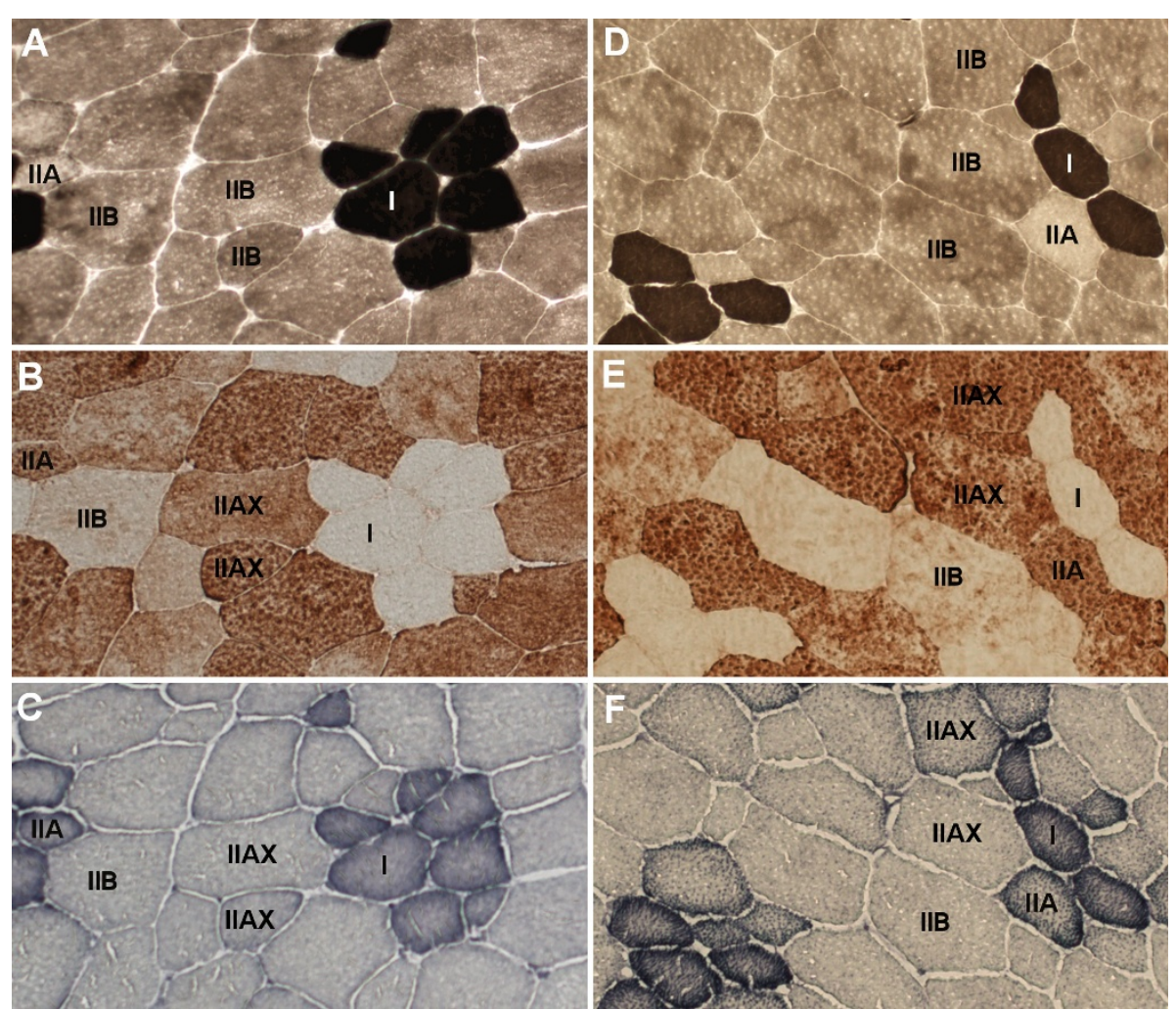

Figure 1 Photomicrographs of serial sections of $m$. longissimus dorsi of carriers $(A, B, C)$ and non-carriers (D, E, F) of the PRKAG3 mutation. Fibre types I, $\| A$, and $\| B$ classified with myosin ATPase ( $\mathrm{pH}$ 4.6) stains (A, D) and fibre type $\| A X$ is classified with immunohistochemical (A4-74) stains (B, E). Note that many type IIB fibres in the myosin ATPase stain were classified as IIAX fibres with the immunohistochemical stain and that some of these IIAX fibres may be pure, IIX or IIBX fibres. Oxidative capacity is evaluated from the NADH tetrazolium reductase stains (C, F). Note that type I fibres have a high staining intensity, whereas staining intensity varies among the subgroups of type II fibres. 
for comparison of values between the carrier and the non-carrier pigs. Means were regarded as significantly different at $P<0.05$. Statistical analyses were carried out using Sigma Stat Statistical Software version 11.0.

\section{Results}

\section{Fibre type composition and mean fibre area}

None of $m$. masseter, $m$. biceps femoris, $m$. semitendinosus or m. longissimus dorsi showed any difference between genotypes in the percentage of type I, IIA and IIB fibres when evaluated from the ATPase stains. Type IIB fibres from the ATPase stain for $m$. longissimus dorsi and $m$. biceps femoris correspond to the sum of IIAX and IIB fibres identified with the immunohistochemical method. A large proportion of type IIB fibres identified from the ATPase stain was seen in $m$. semitendinosus, $m$. longissimus dorsi and $m$. biceps femoris. M. semitendinosus and $m$. longissimus dorsi had a low proportion of type I fibres. A high proportion of type IIA fibres were seen in $m$. masseter (Table 1, 2).

The immunohistochemical method showed that pigs carrying the PRKAG3 mutation had compared to the non-carriers less $(P<0.05)$ percentage of type IIB fibres, in $m$. biceps femoris and in $m$. longssimus dorsi and a higher $(P<0.05)$ percentage of type IIAX fibres in $m$. longissimus dorsi. The mean fibre area of all different fibres types in the carriers was larger $(P<0.05)$ in $m$. biceps femoris and larger $(P<0.05)$ in type I and type IIAX fibres in $m$. longissimus dorsi. In these muscles the relative area of type IIAX fibres was larger $(P<0.05)$ in the carriers and the relative area of type IIB fibres was lower $(P<0.05)$ than in the non-carriers (Table 1$)$.

In all type I fibres the NADH staining intensity was high (Figure 1). Most of the type IIA fibres were stained medium while type IIAX and IIB fibres stained both medium and low in $m$. biceps femoris and $m$. longissimus dorsi. Most real type IIB fibres stained low in both muscles The carriers had, in both $m$. biceps femoris and $m$. longissimus dorsi, a lower percentage $(P<0.05)$ of medium stained type IIAX fibres and a higher $(P<0.05)$ percentage of low stained type IIAX fibres compared to the non-carriers. The staining intensity in type IIB fibres was mainly low, but the carriers had a higher $(P<0.05)$ percentage of medium stained type IIB fibres and a lower $(P<0.05)$ percentage of low stained type IIB fibres in m. longissimus dorsi (Table 1).

Table 1 Fibre characteristics in different muscle groups in carriers and non-carriers of the PRKAG3 mutation

\begin{tabular}{|c|c|c|c|c|}
\hline & \multicolumn{2}{|c|}{ m. biceps femoris } & \multicolumn{2}{|c|}{ m. longissimus dorsi } \\
\hline & Carriers $(n=7)$ & Non-carriers $(n=7)$ & Carriers $(n=7)$ & Non-carriers $(n=7)$ \\
\hline \multicolumn{5}{|l|}{ Fibre type (\%) } \\
\hline । & $27 \pm 1$ & $24 \pm 1$ & $15 \pm 1$ & $13 \pm 1$ \\
\hline$\| \mathrm{A}$ & $7 \pm 1$ & $8 \pm 1$ & $4 \pm 1$ & $2 \pm 1$ \\
\hline$\| A X$ & $37 \pm 2$ & $33 \pm 2$ & $56 \pm 3^{*}$ & $38 \pm 3$ \\
\hline$\| \mathrm{B}$ & $29 \pm 1^{*}$ & $35 \pm 1$ & $25 \pm 2^{*}$ & $47 \pm 3$ \\
\hline \multicolumn{5}{|c|}{ Fibre area $\left(\mu m^{2}\right)$} \\
\hline । & $2471 \pm 194^{*}$ & $1896 \pm 146$ & $2571 \pm 196^{*}$ & $1965 \pm 192$ \\
\hline$\| \mathrm{A}$ & $3346 \pm 330^{*}$ & $2424 \pm 173$ & $2118 \pm 326$ & $1949 \pm 426$ \\
\hline$\| A X$ & $5443 \pm 399^{*}$ & $3352 \pm 276$ & $5088 \pm 430^{*}$ & $3856 \pm 354$ \\
\hline$\| B$ & $7054 \pm 592^{*}$ & $5224 \pm 500$ & $4630 \pm 396$ & $4576 \pm 229$ \\
\hline \multicolumn{5}{|c|}{ Relative fibre area (\%) } \\
\hline । & $15 \pm 2$ & $14 \pm 1$ & $9 \pm 1$ & $6 \pm 1$ \\
\hline$\| \mathrm{A}$ & $5 \pm 1$ & $6 \pm 1$ & $2 \pm 1$ & $2 \pm 1$ \\
\hline$\| A X$ & $41 \pm 2^{*}$ & $33 \pm 2$ & $65 \pm 3^{*}$ & $39 \pm 3$ \\
\hline$\| B$ & $39 \pm 3^{*}$ & $47 \pm 1$ & $25 \pm 3^{*}$ & $53 \pm 4$ \\
\hline \multicolumn{5}{|c|}{ NADH intensity (\%) } \\
\hline I High & $100 \pm 0$ & $100 \pm 0$ & $100 \pm 0$ & $100 \pm 0$ \\
\hline IIA High & $12 \pm 6$ & $12 \pm 5$ & $17 \pm 17$ & $0 \pm 0$ \\
\hline IIA Medium & $88 \pm 6$ & $88 \pm 5$ & $83 \pm 17$ & $100 \pm 0$ \\
\hline IIAX Medium & $57 \pm 4^{*}$ & $92 \pm 3$ & $25 \pm 4^{*}$ & $61 \pm 6$ \\
\hline IIAX Low & $43 \pm 4^{*}$ & $8 \pm 3$ & $75 \pm 4^{*}$ & $39 \pm 6$ \\
\hline IIB Medium & $2 \pm 1$ & $2 \pm 1$ & $13 \pm 3^{*}$ & $4 \pm 2$ \\
\hline IIB Low & $98 \pm 1$ & $98 \pm 1$ & $87 \pm 3^{*}$ & $96 \pm 2$ \\
\hline
\end{tabular}

Fibre type composition was identified with myosin ATPase stains and type I and IIA+ IIX with myosin heavy chain antibodies. $\mathrm{NADH}$-tetrazolium reductase staining intensity was subjectively evaluated as low, medium and high in the different fibre types. Data as means \pm SE. ${ }^{*} P<0.05$ significantly different to non-carriers. 
Table 2 Fibre characteristics in different muscle groups in carriers and non-carriers of the PRKAG3 mutation

\begin{tabular}{|c|c|c|c|c|}
\hline & \multicolumn{2}{|c|}{ m. semitendinosus } & \multicolumn{2}{|c|}{ m. masseter } \\
\hline & Carriers $(n=7)$ & Non-carriers $(n=6)$ & Carriers $(n=7)$ & Non-carriers $(n=7)$ \\
\hline \multicolumn{5}{|c|}{ Fibre type (\%) } \\
\hline I & $16 \pm 1$ & $13 \pm 4$ & $28 \pm 4$ & $32 \pm 4$ \\
\hline$\| \mathrm{A}$ & $4 \pm 1$ & $6 \pm 1$ & $70 \pm 3$ & $67 \pm 4$ \\
\hline$\| B$ & $80 \pm 1$ & $81 \pm 4$ & $2 \pm 0$ & $1 \pm 1$ \\
\hline \multicolumn{5}{|c|}{ Fibre area $\left(\mu m^{2}\right)$} \\
\hline 1 & $2388 \pm 118$ & $2386 \pm 228$ & $1659 \pm 186$ & $2142 \pm 235$ \\
\hline$\| \mathrm{A}$ & $2574 \pm 402$ & $2905 \pm 380$ & $2127 \pm 302$ & $2264 \pm 266$ \\
\hline$\| B$ & $4574 \pm 285$ & $4504 \pm 446$ & $1429 \pm 175$ & $2192 \pm 181$ \\
\hline \multicolumn{5}{|c|}{ Relative fibre area (\%) } \\
\hline । & $9 \pm 1$ & $8 \pm 1$ & $24 \pm 4$ & $31 \pm 4$ \\
\hline$\| A$ & $3 \pm 1$ & $4 \pm 0$ & $74 \pm 4$ & $68 \pm 4$ \\
\hline$\| B$ & $88 \pm 1$ & $88 \pm 1$ & $1 \pm 0$ & $2 \pm 1$ \\
\hline
\end{tabular}

Fibre type composition was identified using myosin ATPase stains.

Data as means $\pm \mathrm{SE}$.

There were no genotype differences in fibre type area and relative fibre type area in $m$. semitendinosus and m. masseter (Table 2).

\section{Enzyme activities}

The CS, HAD, LDH, HK and phosphorylase activities of $m$. longissimus dorsi, $m$. biceps femoris, and $m$. semitendinosus in the carriers and the non-carriers of the PRKAG3 mutation are presented in Table 3. The CS activity was higher $(P<0.05)$ in the carriers of the PRKAG3 mutation than in the non-carriers only in $m$. longissimus dorsi, and there was no difference between genotypes regarding HAD activity in any of the muscles. The activity of LDH was lower $(P<0.05)$ in the carriers of the PRKAG3 mutation in $m$. longissimus dorsi and in $m$. semitendinosus than in the noncarriers. In all muscles the activity of HK was higher $(P<0.05)$ in the carriers and the activity of phosphorylase was higher $(P<0.05)$ in $m$. biceps femoris and $m$. longissimus dorsi in the carriers than in the noncarriers.

\section{Glycogen analyses}

Pigs carrying the PRKAG3 mutation had in $m$. longissimus dorsi, $m$. biceps femoris and $m$. semitendinosus a higher $(P<0.05)$ concentration of glycogen (Table 3$)$ than the non-carriers. In $m$. masseter the glycogen concentration was also higher $(P<0.05)$ in the carriers $(268 \pm 26 \mathrm{mmol} / \mathrm{kg})$ than in the non-carriers $(166 \pm 19$ $\mathrm{mmol} / \mathrm{kg})$.

\section{Discussion}

The main new finding of this study is, that after exercise training the PRKAG3 mutation influences metabolic and fibre characteristics to a varying degree among muscles with different functions. Fibre type composition and the physical activity level of the muscle are factors that may contribute to the differences seen in glycogen content and enzyme activities between muscles. In agreement with earlier studies on untrained pigs, the pigs carrying the PRKAG3 mutation had in comparison to the noncarriers, higher content of glycogen in both $m$. longissimus dorsi and in $m$. biceps femoris $[1,18,19]$. Previous

Table 3 Enzyme activities and glycogen concentration in different muscle groups in carriers and non-carriers of the PRKAG3 mutation

\begin{tabular}{lcccccc}
\hline & \multicolumn{2}{c}{$\boldsymbol{m}$. longissimus dorsi } & \multicolumn{2}{c}{ m. semitendinosus } & \multicolumn{2}{c}{ m. biceps femoris } \\
\cline { 2 - 7 } & Carriers $(\mathbf{n}=\mathbf{6})$ & Non-carriers $(\mathbf{n}=\mathbf{6})$ & Carriers $(\mathbf{n}=\mathbf{6})$ & Non-carriers $(\mathbf{n}=\mathbf{7})$ & Carriers $(\mathbf{n}=\mathbf{7})$ & Non-carriers $(\mathbf{n}=7)$ \\
\hline CS & $20 \pm 3^{*}$ & $8 \pm 5$ & $15 \pm 1$ & $13 \pm 2$ & $19 \pm 2$ & $20 \pm 1$ \\
HAD & $24 \pm 1$ & $24 \pm 2$ & $26 \pm 2$ & $26 \pm 2$ & $31 \pm 3$ & $29 \pm 3$ \\
HK & $8 \pm 1^{*}$ & $3 \pm 2$ & $7 \pm 1^{*}$ & $4 \pm 1$ & $8 \pm 1^{*}$ & $5 \pm 1$ \\
Phosphorylase & $18 \pm 2^{*}$ & $15 \pm 2$ & $17 \pm 2$ & $15 \pm 3$ & $16 \pm 2^{*}$ & $11 \pm 1$ \\
LDH & $2778 \pm 328^{*}$ & $3199 \pm 134$ & $2929 \pm 187^{*}$ & $3255 \pm 203$ & $2474 \pm 219$ & $2561 \pm 179$ \\
Glycogen & $725 \pm 46^{*}$ & $458 \pm 32$ & $600 \pm 49^{*}$ & $349 \pm 18$ & $681 \pm 42^{*}$ & $420 \pm 28$ \\
\hline
\end{tabular}

Data are expressed as $\mathrm{mmol} / \mathrm{kg} / \mathrm{min}$ for citrate synthase (CS), 3-hydroxyacyl-CoA (HAD), hexokinase (HK), phosphorylase, lactate dehydrogenase (LDH) and in $\mathrm{mmol} / \mathrm{kg}$ for glycogen concentration.

Data as means $\pm \mathrm{SE}$. ${ }^{*} P<0.05$ significantly different from non-carriers. 
studies have shown that the mutation does mainly affect white glycolytic muscles such as $m$. longissimus dorsi and has no effect on a red muscle such as $m$. semispinalis capitis [4]. M. masseter is considered to be a red muscle based on a high CS activity and low glycolytic potential whereas m.longissimus is a glycolytic muscle based on a low CS activity and high glycolytic potential [20]. M. semitendinosus of non-carriers had similar metabolic and fibre characteristics as seen in $m$. longissimus dorsi and is thus considered to be a white glycolytic muscle. As expected the carriers of the PRKAG3 mutation had higher glycogen content also in this muscle. The fact that the total glycogen content seemed to be somewhat lower in $m$. semitendinosus than in $m$. longissimus dorsi is in agreement with earlier observations of non-carriers of the PRKAG3 mutation [10]. Notable was that the carriers of the PRKAG3 mutation had higher glycogen content than the non-carriers also in $m$. masseter, which is considered to be a red oxidative muscle. However, as seen in the present study, some glycolytic type II fibres exist in this muscle. These may be influenced by the mutation, resulting in overall higher glycogen content. The higher synthesis of glycogen in the muscles of the carriers of the PRKAG3 mutation is likely related to a higher capacity for phosphorylation of glucose as indicated by the higher HK activity observed in the muscles. The PRKAG3 mutation may also have an effect on glycogenolysis in association with high muscle glycogen storage as indicated by the higher phosphorylase activity found in both $m$. longissimus dorsi and $m$. biceps femoris in the carriers. The higher phosphorylase and HK activity observed in $m$. biceps femoris of the exercise trained carriers is in agreement with results on young untrained carriers [14]. This indicates that the PRKAG3 mutation has a great influence on these enzymes and may suggest that the carriers of the mutation have an increased glycogen turnover. The increased oxidative capacity (indicated by the higher CS activity) and the decreased glycolytic capacity (indicated by lower LDH activity) in $m$. longissimus dorsi of the carriers of the PRKAG3 mutation, is also in agreement with earlier studies of untrained pigs [4,9]. In a previous study the HAD activity was higher in m.longissimus dorsi [4] but this was not seen in any of the muscles in the present study. A study with transgenic mice models showed that mice with a chronically AMPK-activating mutation caused a shift from fibre type B to IIA/X fibres [21]. These mice had higher activity of CS and increased hexokinase protein expression regardless if they had exercised or not. AMPK signalling was suggested to play an important role for transforming skeletal muscle fibre types as well as for increasing hexokinase II protein expression and oxidative capacity. These findings are in agreement with effects of the PRKAG3 mutation on muscle characteristics in the present study especially in $m$. longissimus dorsi. Studies on transgenic mice (Tg-Prkag ${ }^{225 \mathrm{Q}}$ ) have shown that the PRKAG3 mutation is associated with a greater basal AMPK activity [22]. Previous studies of fibre characteristics in $m$. longissimus dorsi in pigs that carry the PRKAG3 mutation indicate that alterations may occur in the subgroups of type II fibres $[4,23]$. This is also in agreement with the findings of the present study. Notable was that the carriers of the PRKAG3 mutation had less IIB fibres, not only in $m$. longissimus dorsi, but also in m. biceps femoris, compared to non-carriers. The fact that the oxidative capacity evaluated by the CS activity in the present study did not differ between genotypes in $m$. biceps femoris but differed in $m$. longissimus dorsi, may be related to these muscles being differently involved during locomotion [10]. It has earlier been indicated that adaptations to training differ between muscles [10]. Endurance trained pigs had in comparison to non-trained pigs an increased oxidative capacity and a higher glycogen content in $m$. biceps femoris, but no differences were seen in $m$. longissimus dorsi and in $m$. semitendinosus, muscles thus considered to be less involved during training on a treadmill [10].

In both genotypes training adaptations in the fibres of $m$. biceps femoris may have caused a similar oxidative capacity in response to the increased energy demand during locomotion. A previous study of pigs has shown that glycogen is lowered in both genotypes in type I, IIA and in some IIB fibres in $m$. biceps femoris during the same type of exercise as used in this study, which indicates that these fibres have been recruited [19]. Adaptations to exercise training in this muscle may have decreased the effects of the PRKAG3 mutation on muscle metabolic and contractile properties.

The carriers had less type IIB fibres in $m$. longissimus dorsi which indicates that one effect of the PRKAG3 mutation may be associated with transformation of type IIB towards type IIX and IIA fibres, as carriers also had more type IIAX fibres. The muscle fibres that are classified as MHCIIAX may be a mixture of pure IIX and/or hybrid IIA+IIX and IIX+IIB as the antibody A4-74 identifies both IIA and IIX fibres [24,25]. Transition of myosin heavy chains is said to follow a sequential, yet reversible, pathway: I↔IIA $\leftrightarrow$ IIAX $\leftrightarrow$ IIX $\leftrightarrow$ IIB $[26,27]$. Interestingly, genetic selection for growth performance in pigs, shifts fibre type towards type IIB fibres $[28,29]$ whereas endurance exercise training has been shown to shift the fibre type towards type IIA in rats [30] and in man [31]. Studies in pigs also indicate that fibre type shifts from type IIB to IIA may occur with training [32,33]. Oxidative capacity is known to increase with training and among fibre types oxidative metabolism is high in type I fibres and decreases in the rank order 
from type I to type IIA to type IIX to type IIB fibres [34]. Intensive selection for a higher meat content and lean muscle growth in modern pigs has not only caused shifts in contractile fibre types, but also induced a change in muscle metabolism towards a more glycolytic and less oxidative fibre type [35]. In contrast, the PRKAG3 mutation has been shown to decrease IIB and increase IIA and IIX mRNA expression, which also implies that the genotype promotes a more oxidative phenotype [23]. The changes seen in muscle characteristics in the carriers with the PRKAG3 mutation thus resemble those seen when muscles in pigs adapt to an increased physical activity level. In rabbits contractile activity induces a fast-to-slow and glycolytic-to-oxidative fibre transition in skeletal muscle [36]. In the present study the pigs with the mutation in the $\gamma$-subunit of AMPK seem to have developed a more oxidative phenotype independent of contractile activity. This is supported by the higher CS activity and the higher oxidative capacity of type IIB muscle fibre types according to the NADH-tetrazolium reductase staining found in $m$. longissimus dorsi of the carriers. Notable, many type IIAX fibres in the carriers were classified as having a low oxidative capacity. However, these IIAX fibres in the carriers probably also had an overall higher oxidative capacity as they were larger in size. As seen from Figure 1, the staining intensity for NADH-tetrazolium reductase is usually homogeneous within a fibre, but more intense at the periphery due to a higher density of mitochondria there.

The muscle fibre composition of $m$. masseter, $m$. semitendinosus, $m$. biceps femoris and $m$. longissimus dorsi identified according to the ATPase stains is in good agreement with earlier studies [10,37]. If differences among subgroups of type II fibres (including hybrids) also occurred in $m$. semitendinosus and $m$. masseter between the two genotypes is not known, since only the ATPase staining technique was used to identify fibre types in these muscles. If fibre types had been identified only from the ATPase stains in $m$. longissimus dorsi and $m$. biceps femoris, no changes in subgroups of type II fibres between the two genotypes would have been revealed. This clearly shows that the use of antibodies against the different myosin heavy chains will give a more detailed picture of the fibre type composition in a muscle. When pure IIX and hybrid IIA+IIX and IIX+IIB fibres cannot be detected alterations in muscle fibre types might be overlooked.

The MHCIIB isoform was previously said to exist only in small animals such as mouse, rat, guinea pig and rabbit $[38,12]$. However, studies have shown that large animals i.e. pigs and llamas do exhibit MHCIIB fibres and mostly in glycolytic muscles $[34,39,40]$. In fact the $m$. longissimus dorsi has been shown to contain $51 \%$ of type MHCIIB in pigs [41]. This is in good agreement with $47 \%$ type IIB fibres observed in the $m$. longissimus dorsi of non-carriers in the present study. As seen from Figure 1 the NADH-staining intensity showed marked differences in oxidative capacity among the fibre types and as expected type IIB fibres had mainly a low oxidative capacity. Whether type IIAX fibres with low staining intensity for oxidative capacity correspond to pure type IIX and/or hybrid type IIX + IIB needs to be investigated in future studies using antibodies that can separate MHCIIA and MHCIIX fibres.

\section{Conclusions}

In exercise-trained pigs, the PRKAG3 mutation influences muscle characteristics and promotes an oxidative phenotype to a varying degree among muscles with different functions. The present results show that the carriers of the PRKAG3 mutation are of interest not only in meat science, but also as a large animal model for in vivo studies of the carbohydrate metabolism.

\section{Acknowledgements \\ The financial support of The Swedish Research Council for Environment, Agricultural Sciences and Spatial Planning is gratefully acknowledged.}

\section{Authors' contributions}

All authors participated in the design of the study and the collection of samples. AG performed laboratory analyses and statistical calculations. AG and BEG have interpreted the data and drafted the manuscript. All authors read and approved the final manuscript.

\section{Competing interests}

The authors declare that they have no competing interests.

Received: 27 October 2010 Accepted: 24 March 2011

Published: 24 March 2011

\section{References}

1. Enfält AC, Lundström K, Karlsson A, Hansson I: Estimated frequency of the $\mathrm{RN}^{-}$allele in Swedish Hampshire pigs and comparison of glycolytic potential, carcass composition, and technological meat quality among Swedish Hampshire, Landrace, and Yorkshire pigs. J Anim Sci 1997, 75:2924-2935.

2. Le Roy P, Elsen JM, Caritez JC, Talmant A, Juin H, Sellier P, Monin G: Comparison between the three porcine RN genotypes for growth, carcass composition and meat quality traits. Genet Sel Evol 2000, 32:165-186.

3. Milan D, Jeon JT, Looft C, Amarger $\mathrm{V}$, Robic A, Thelander M, RogelGaillard C, Paul S, lannuccelli N, Rask L, Ronne H, Lundstrom K, Reinsch N, Gellin J, Kalm E, Roy PL, Chardon P. Andersson L: A mutation in PRKAG3 associated with excess glycogen content in pig skeletal muscle. Science 2000, 288:1248-1251.

4. Lebret B, Le Roy P, Monin G, Lefaucheur L, Caritez JC, Talmant A, Elsen JM, Sellier $P$ : Influence of the three RN genotypes on chemical composition, enzyme activities, and myofiber characteristics of porcine skeletal muscle. J Anim Sci 1999, 77:1482-1489.

5. Hardie DG, Carling D: The AMP-activated protein kinase: Fuel gauge of the mammalian cell? Eur J Biochem 1997, 246:259-273.

6. Winder WW: Energy-sensing and signaling by AMP-actvated protein kinase in skeletal muscle. J Appl Physiol 2001, 91:1017-1028.

7. Mu J, Brozinick JTJ, Valladares O, Bucan M, Birnbaum MJ: A role for AMPactivated protein kinase in contraction-and hypoxia-regulated glucose transport in skeletal muscle. Mol Cell 2001, 7:1085-1094. 
8. Wright DC, Hucker KA, Holloszy JO, Han DH: Ca2+ and AMPK both mediate stimulation of glucose transport by muscle contractions. Diabetes 2004, 53:330-335.

9. Estrade M, Ayoub S, Talmant A, Monin G: Enzyme activities of glycogen metabolism and mitochondrial characteristics in muscles of RN-carrier pigs (Sus scrofa domesticus). Comp Biochem Physiol Biochem Mol Biol 1994, 108:295-301.

10. Essén-Gustavsson B, Lindholm A, Persson S: The effect of exercise and anabolic steroids on enzyme activities and fibre composition in skeletal muscle of pigs. Proceedings of fifth Meeting of the Academic Society for Large Animal Veterinary Medicine: 1980; Glasgow 1980, 44-54

11. Essén-Gustavsson B, Lindholm A: Influence of exercise on muscle metabolic characteristics in pigs and backfat thickness at slaughter. Proceedings of fifth International Conference on Production Disease in Farm Animals: 1983; Uppsala 1983, 356-362.

12. Schiaffino S, Gorza L, Sartore S, Saggin L, Ausoni S, Vianello M, Gundersen K, Lømo T: Three myosin heavy chain isoforms in type 2 skeletal muscle fibers. J Muscle Res Cell Motil 1989, 10:197-205.

13. Schiaffino S: Fibre types in skeletal muscle: a personal account. Acta Physiol 2010, 199:451-63.

14. Granlund A, Kotova O, Benziane B, Galuska D, Jensen-Waern M, Chibalin AV, Essén-Gustavsson B: Effects of exercise on muscle glycogen synthesis signalling and enzyme activities in pigs carrying the PRKAG3 mutation. Exp Physiol 2010, 95:541-549.

15. Brooke $\mathrm{MH}$, Kaiser KK: Muscle fiber types: how many and what kind? Arch Neurol 1970, 23:369-379.

16. Novikoff AB, Shin W, Drucher J: Mitochondrial localization of oxidative enzymes: Staining results with two tetrazolium salt. J Biophys Biochem Cytol 1961, 9:47-61

17. Lowry OH, Passoneau JV: A flexible system of enzymatic analysis. Academic Press New York; 1973, 1-291.

18. Monin G, Brard C, Vernin P, Naveau J: Effects of the $\mathrm{RN}^{-}$gene on some traits of muscle and liver in pigs. Proceedings of 38th International Congress of Meat Science Technology: 1992; Clermont-Ferrand, France 3:391-394.

19. Essén-Gustavsson $B$, Jensen-Waern $M$, Jonasson $R$, Andersson L: Effect of exercise on proglycogen and macroglycogen content in skeletal muscles of pigs with the Rendement Napole mutation. AJVR 2005, 66:1197-1201.

20. Monin G, Mejenes-Quijano A, Talmant A: Influence of breed and muscle metabolic type on muscle glycolytic potential and meat $\mathrm{pH}$ in pigs. Meat Sci 1987, 20:149-158.

21. Röckl KSC, Hirschman MF, Brandauer J, Fujii N, Witters LA, Goodyear LJ: Skeletal muscle adaptation to exercise training: AMP-activated protein kinase mediates muscle fiber type shift. Diabetes 2007, 56:2062-2069.

22. Barnes BR, Marklund S, Steiler T, Walter M, Hiälm G, Amarger V, Mahlapuu M, Leng Y, Johansson C, Galuska D, Lindgren K, Åbrink M, Stapleton D, Zierath JR, Andersson L: The 5'-AMP-activated protein kinase gamma 3 isoform has a key role in carbohydrate and lipid metabolism in glycolytic skeletal muscle. J Biol Chem 2004, 37:38441-38447.

23. Park SK, Gunawan AM, Scheffler TL, Grant AL, Gerrard DE: Myosin heavy chain isoform content and energy metabolism can be uncoupled in pig skeletal muscle. J Anim Sci 2009, 87:522-531.

24. Graziotti GH, Delhon G, Rios CM, Rivero JL: Fiber muscle types in adult female pigs as determined by combinating histochemical and immunohistochemical methods. Rev Chil Anat 2001, 19:167-73.

25. Fazarinc G: Enzyme-immunohistochemical aspects of muscle fiber type classification in mammals. Slov Vet Res 2009, 46:61-70.

26. Schiaffino S, Reggiano C: Myosin isoforms in mammalian skeletal muscle. J Appl Physiol 1994, 77:493-501.

27. Pette D, Staron RS: Myosin isoforms, muscle fiber types, and transitions. Microsc Res Tech 2000, 50:500-509.

28. Solomon MB, West RL: Profile of fiber types in muscles from wild pigs native to the United States. Meat Sci 1985, 13:247-254.

29. Weiler U, Appell HJ, Kremser M, Hofläcker S, Claus R: Consequences of selection on muscle composition. A comparative study on gracilis muscle in wild and domestic pigs. Anat Histol Embryol 1995, 24:77-80.

30. Green HJ, Klug GA, Reichmann H, Seedorf U, Wiehrer W, Pette D: Exerciseinduced fibre type transitions with regard to myosin, parvalbumin, and sarcoplasmic reticulum in muscles of the rat. Pflugers Arch 1984, 400:432-438.
31. Baumann $H$, Jäggi $M$, Soland $F$, Howald $H$, Schaub MC: Exercise training induces transitions of myosin isoform subunits within histochemically typed human muscle fibres. Pflugers Arch 1987, 409:349-360.

32. Essén-Gustavsson B: Muscle-fibre characteristics in pigs and relationships to meat-quality parameters. In Pork quality: Genetic and metabolic factors. Edited by: Poulanne E, Deymer DI, Ruusunen M, Ellis S. CAB International, Wallingford, UK; 1993:140.

33. Petersen JS, Henckel P, Oksbjerg N, Sorensen MT: Adaptations in muscle fibre characteristics induced by physical activity in pigs. J Anim Sci 1998, 66:733-740.

34. Lefaucheur L, Ecolan P, Plantard L, Gueguen N: New insights into muscle fiber types in the pig. J Histochem Cytochem 2002, 50:719-730.

35. Lefaucheur $L$ : A second look into fibre typing - Relation to meat quality. Meat Sci 2010, 84:257-270.

36. Green HJ, Düsterhöft $S$, Dux L, Pette D: Metabolite patterns related to exhaustion, recovery and transformation of chronically stimulated rabbit fast-twitch muscle. Pflugers Arch 1992, 420:359-366.

37. Karlström K: Cappilary supply, fibre type composition and enzymatic profile of equine, bovine and porcine locomotor and nonlocomotor muscles. PhD thesis Swedish University of Agricultural Sciences, Uppsala; 1995.

38. Bär A, Simoneau JA, Pette D: Altered expression of myosin light chain isoforms in chronically stimulated fast-twitch muscle of the rat. Eur $J$ Biochem 1989, 178:591-594.

39. Lefaucheur L, Hoffman RK, Gerrard DE, Okamura CS, Rubinstein N, Kelly A: Evidence for three adult fast myosin heavy chain isoforms in type II skeletal muscle fibers in pigs. J Anim Sci 1998, 76:1584-1593.

40. Graziotti GH, Rios CM, Rivero JLL: Evidence for three fast myosin heavy chain isoforms in type II skeletal muscle fibers in the adult llama (Lama glama). J Histochem Cytochem 2001, 49:1033-1044.

41. Toniolo L, Patruno M, Maccatrozzo L, Pellegrino MA, Canepari M, Rossi R, D'Antona G, Bottinelli R, Reggiani C, Mascarello F: Fast fibres in a large animal: fibre types, contractile properties and myosin expression in pig skeletal muscles. J Exp Biol 2004, 207:1875-86.

doi:10.1186/1751-0147-53-20

Cite this article as: Granlund et al:: The influence of the PRKAG3 mutation on glycogen, enzyme activities and fibre types in different skeletal muscles of exercise trained pigs. Acta Veterinaria Scandinavica 2011 53:20.

\section{Submit your next manuscript to BioMed Central and take full advantage of:}

- Convenient online submission

- Thorough peer review

- No space constraints or color figure charges

- Immediate publication on acceptance

- Inclusion in PubMed, CAS, Scopus and Google Scholar

- Research which is freely available for redistribution

Submit your manuscript at www.biomedcentral.com/submit
C Biomed Central 